\title{
Hypothalamic resistin induces hepatic insulin resistance
}

\author{
Evan D. Muse,1,2,3 Tony K.T. Lam,1,2,3 Philipp E. Scherer, ${ }^{2,3,4}$ and Luciano Rossetti1,2,3 \\ 1Department of Molecular Pharmacology, ${ }^{2}$ Department of Medicine, ${ }^{3}$ Diabetes Research and Training Center, and ${ }^{4}$ Department of Cell Biology, \\ Albert Einstein College of Medicine of Yeshiva University, New York, New York, USA.
}

\begin{abstract}
Circulating resistin stimulates endogenous glucose production (GP). Here, we report that bi-directional changes in hypothalamic resistin action have dramatic effects on GP and proinflammatory cytokine expression in the liver. The infusion of either resistin or an active cysteine mutant in the third cerebral ventricle (icv) or in the mediobasal hypothalamus stimulated GP independent of changes in circulating levels of glucoregulatory hormones. Conversely, central antagonism of resistin action markedly diminished the ability of circulating resistin to enhance GP. We also report that centrally mediated mechanisms partially control resistin-induced expression of TNF- $\alpha$, IL-6, and SOCS-3 in the liver. These results unveil what we believe to be a novel site of action of resistin on GP and inflammation and suggest that hypothalamic resistin action can contribute to hyperglycemia in type 2 diabetes mellitus.
\end{abstract}

\section{Introduction}

The increased incidence of type 2 diabetes mellitus is closely correlated with the increased prevalence of obesity. The figures from the latest National Health and Nutrition Examination Survey (NHANES) show that two-thirds of the adult US population can be classified as overweight or obese, and most alarmingly the prevalence of obesity among children continues to rise $(1,2)$. Thus, it is becoming of even greater importance to better understand the elusive etiology and progression from obesity to type 2 diabetes mellitus $(3,4)$. Although some evidence, such as increased circulating free fatty acids (5) and decreased adiponectin $(6,7)$, link the metabolic milieu that accompanies increased adiposity to insulin resistance, the role of adipose tissue as both an inflammatory mediator and endocrine organ has recently increased in interest (8-10). Of the described adipose-derived factors, also known as adipokines, resistin seems to assert its effects on both inflammatory and insulin signaling pathways (11).

Resistin, also known as found in inflammatory zone 3 (FIZZ3) and adipocyte-specific secretory factor, is a recently discovered adipokine that belongs to a family of small, cysteine-rich secreted proteins (12-14). While resistin is secreted solely from adipose tissue in rodents, it is mainly derived from monocytes and macrophage in humans $(15,16)$. We have previously shown in rodents that the plasma resistin concentration is increased after high-fat feeding and that this increase is the primary cause of hepatic insulin resistance (17). Additional animal studies have highlighted the ability of resistin to induce hepatic insulin resistance after both acute and chronic administration (12, 18-20). Human studies have since linked resistin to increased

Nonstandard abbreviations used: ACC1, acetyl-CoA carboxylase 1; aCSF, artificial cerebrospinal fluid; AMPK, AMP-activated protein kinase; Con Ab, control Ab; cys, cysteine mutant of resistin; FAS, fatty acid synthase; G6Pase, glucose-6-phosphatase; GP, glucose production; GSK3 $\beta$, glycogen synthase kinase $3 \beta$; I $\kappa$ B, inhibitor of NF-кB; IKK, IкB kinase; IH, intrahypothalamic; MBH, mediobasal hypothalamus; p-, phosphorylated; PEP, phosphoenolpyruvate; PEPCK, PEP carboxykinase; PGC-1 $\alpha$, PPAR $\gamma$ coactivator $1 \alpha$; Rs Ab, anti-mouse resistin Ab; SCD1, steroyl-CoA decarboxylase 1; UDP-glucose, uridine diphospho-glucose.

Conflict of interest: The authors have declared that no conflict of interest exists. Citation for this article: J. Clin. Invest. 117:1670-1678 (2007). doi:10.1172/JCI30440. central adiposity (21), insulin resistance (22), atherosclerosis, and inflammation $(23,24)$. The fact that human resistin is produced by monocytes and macrophages provides greater affirmation of the immune system's involvement in resistin's role in metabolic diseases (25). It is widely accepted that inflammation leads to insulin resistance (26), and the important roles of TNF- $\alpha$ $(27,28)$, SOCS-3 $(29,30)$, STAT3 (31), and inhibitor of NF-кB (IКB) kinase (IKK) (32) as signaling mediators of hepatic glucose homeostasis in this inflammation/insulin resistance axis have been reported (9).

Recently, it has been shown that resistin mRNA and protein are both present in mouse hypothalamus $(33,34)$ and that resistin activates a certain subset of hypothalamic neurons in vitro (35). With work from our laboratory as well as by others highlighting the importance of the brain-liver circuit in controlling hepatic glucose homeostasis in response to hypothalamically initiated hormonal (i.e., insulin and leptin) (36-38) and nutritional (i.e., FFA and glucose) signals (39-41), resistin also seemed a likely candidate to act via hypothalamic pathways. Since the effects are at least in part mediated via interactions with receptors within the CNS, it is postulated herein that resistin regulates glucose fluxes and signaling in the liver both directly via hepatic effects and indirectly through a central (hypothalamic) site of action (Figure 1A). In this study, we investigated whether the brain also plays a role in mediating the diabetogenic effects of physiological hyperresistinemia and identify potential mechanisms by which resistin modulates hepatic glucose fluxes. To determine whether an increase in resistin made available to the CNS would modulate peripheral insulin action, we made use of the hyperinsulinemic-euglycemic clamp combined with icv and mediobasal hypothalamus (MBH) infusions of recombinant resistin. Furthermore, MBH administration of a specific anti-mouse resistin antibody (Rs Ab) was utilized to assess what contribution central resistin action made to the effect of circulating resistin on whole-body glucose homeostasis. Lastly, we aimed to further investigate the complex relationship between inflammation and insulin resistance in mediating resistin's effects on glucose fluxes. The adipose-derived hormone resistin rapidly stimulates glucose production (GP) and induces hepatic insulin resistance in rodents (17-20). 

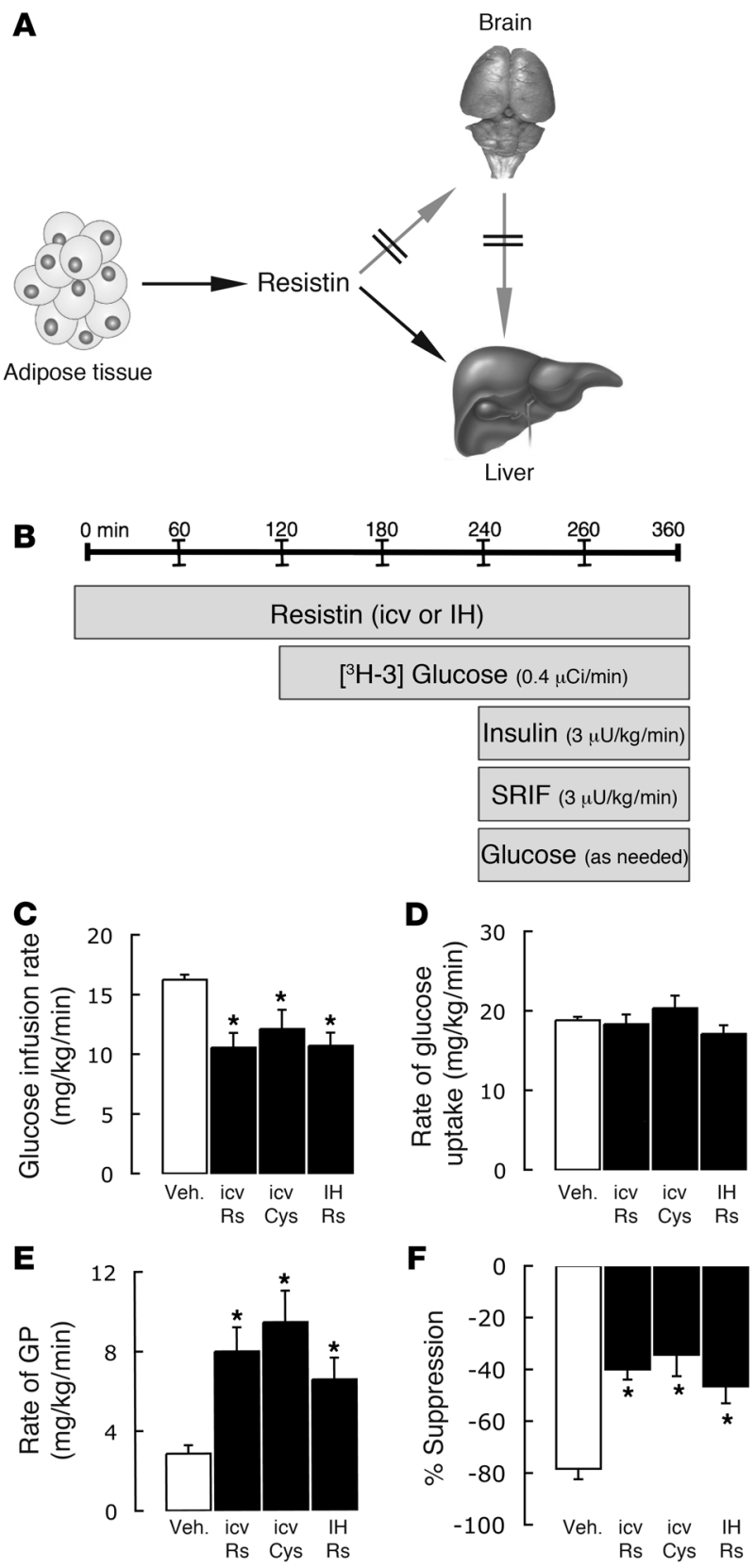

\section{Results}

To test our hypothesis, we first examined whether a primary increase in the central or hypothalamic availability of resistin per se is sufficient to stimulate GP and generate hepatic insulin resistance. We next examined the systemic effects of hypothalamic resistin action by inducing a physiological increase in the circulating levels of resistin while selectively negating resistin's central effects through the infusion of a specific anti-resistin $\mathrm{Ab}$.

Central administration of recombinant resistin induces hepatic insulin resistance. To investigate the central effects of resistin independent of its pleiotropic systemic actions, we examined whether the central administration of recombinant resistin acutely regulates hepatic insulin action. To this end, 3 groups of conscious rats received icv infusions of recombinant mouse resistin, a biologically active cysteine mutant of resistin (cys), or vehicle (Figure 1B

\section{Figure 1}

Central administration of recombinant resistin induces hepatic insulin resistance in rats. (A) Mechanisms of resistin action on hepatic GP. Increased circulating levels of resistin lead to impaired hepatic insulin action, though whether this is mediated in part via pathways initiated in the hypothalamus is unknown. Here we investigate this "indirect" pathway; arrows interrupted by double bars indicate our attempt to block pathway using Rs Ab. (B) Experimental design for hyperinsulinemic-euglycemic clamp studies. Resistin infusion studies lasted 360 minutes. Recombinant mouse resistin or aCSF was infused into Sprague-Dawley rats via icv ( $300 \mathrm{ng}$ total; $8 \mathrm{ng} / \mu \mathrm{l}$ at $5 \mu \mathrm{l} / \mathrm{h}$ ) or $\mathrm{IH}$ (16 $\mathrm{ng}$ total; $8 \mathrm{ng} / \mu \mathrm{l}$ at $0.33 \mu \mathrm{l} / \mathrm{h})$ cannulae. At 120 minutes, rats received a primed-constant infusion of $\left[{ }^{3} \mathrm{H}-3\right]$ glucose $(0.4 \mu \mathrm{Ci} / \mathrm{min})$. Hyperinsulinemic-euglycemic pancreatic/insulin clamp was initiated at 240 minutes, with a constant infusion of insulin ( $3 \mathrm{mU} / \mathrm{kg} / \mathrm{min})$ and somatostatin ( $3 \mu \mathrm{g} / \mathrm{kg} / \mathrm{min}$ ); infusion of $10 \%$ glucose solution was periodically adjusted to maintain steady plasma glucose concentration. SRIF, somatostatin. (C) Glucose infusion rates necessary to maintain euglycemia in the presence of hyperinsulinemia ( $3 \mathrm{mU} / \mathrm{kg} / \mathrm{min}$ ) were significantly lower in resistin-treated groups compared with vehicle. (D) Rates of glucose uptake were unaffected by central infusion of resistin. (E) Rates of endogenous GP for resistin-infused animals were nearly 2-fold greater than those of vehicle. (F) Percentage suppression of endogenous GP induced by insulin infusion ( $3 \mathrm{mU} / \mathrm{kg} / \mathrm{min}$ ), a clear readout of wholebody insulin sensitivity, was reduced $50 \%$ in resistin-treated groups compared with vehicle. ${ }^{\star} P<0.05$ compared with vehicle (Veh.).

and Supplemental Table 1; supplemental material available online with this article; doi:10.1172/JCI30440DS1). The cys used in these studies has been shown to potently stimulate fluxes in liver glucose (42). The icv infusion of resistin or cys moderately increased the plasma glucose and insulin levels (Supplemental Table 1). Insulin action was assessed by a combination of icv infusions using the hyperinsulinemic-euglycemic clamp technique (Figure 1B). As expected, in the presence of the approximately 3 -fold increase in the circulating insulin levels (using the clamp technique; Supplemental Table 1), glucose had to be infused in order to maintain euglycemia in icv vehicle-infused animals $(15.6 \pm 0.4 \mathrm{mg} / \mathrm{kg} / \mathrm{min})$. By contrast, following icv infusions of resistin or cys, glucose was infused at a much lower rate of approximately $10 \mathrm{mg} / \mathrm{kg} / \mathrm{min}$ in order to prevent hypoglycemia (Figure 1C). It should be noted that the plasma insulin and glucagon concentrations (data not shown) were similar in the 3 groups (Supplemental Table 1). Thus, central administration of resistin diminished hepatic insulin action on glucose homeostasis.

We next examined the potential mechanism(s) by which icv resistin impaired whole-body insulin action. We assessed glucose kinetics by tracer dilution methodology in order to establish whether the decreased rate of glucose infusion induced by icv resistin or cys was due to either a decrease in glucose uptake or a stimulation of endogenous GP. The rates of glucose utilization (Figure 1D) were not significantly affected by either icv resistin $(19.6 \pm 0.5 \mathrm{mg} / \mathrm{kg} / \mathrm{min})$ or cys $(20.4 \pm 1.0 \mathrm{mg} / \mathrm{kg} / \mathrm{min})$ compared with vehicle $(19.8 \pm 0.3 \mathrm{mg} / \mathrm{kg} / \mathrm{min})$. Conversely, icv resistin and cys markedly increased the rate of GP and therefore impaired the suppressive effect of circulating hyperinsulinemia on the rate of GP (Figure 1, E and F). icv vehicle infusion was associated with a marked $(-75 \%)$ decline in the rate of GP from basal levels (Figure $1 \mathrm{~F})$. However, icv resistin and cys lead to a modest ( $40 \%)$ decline in the rate of hepatic GP compared with basal rates (Figure 1F). These changes in glucose output occurred in the presence of similar plasma insulin levels $(\sim 75 \mu \mathrm{U} / \mathrm{ml}$; Supplemental Table 1$)$ and 

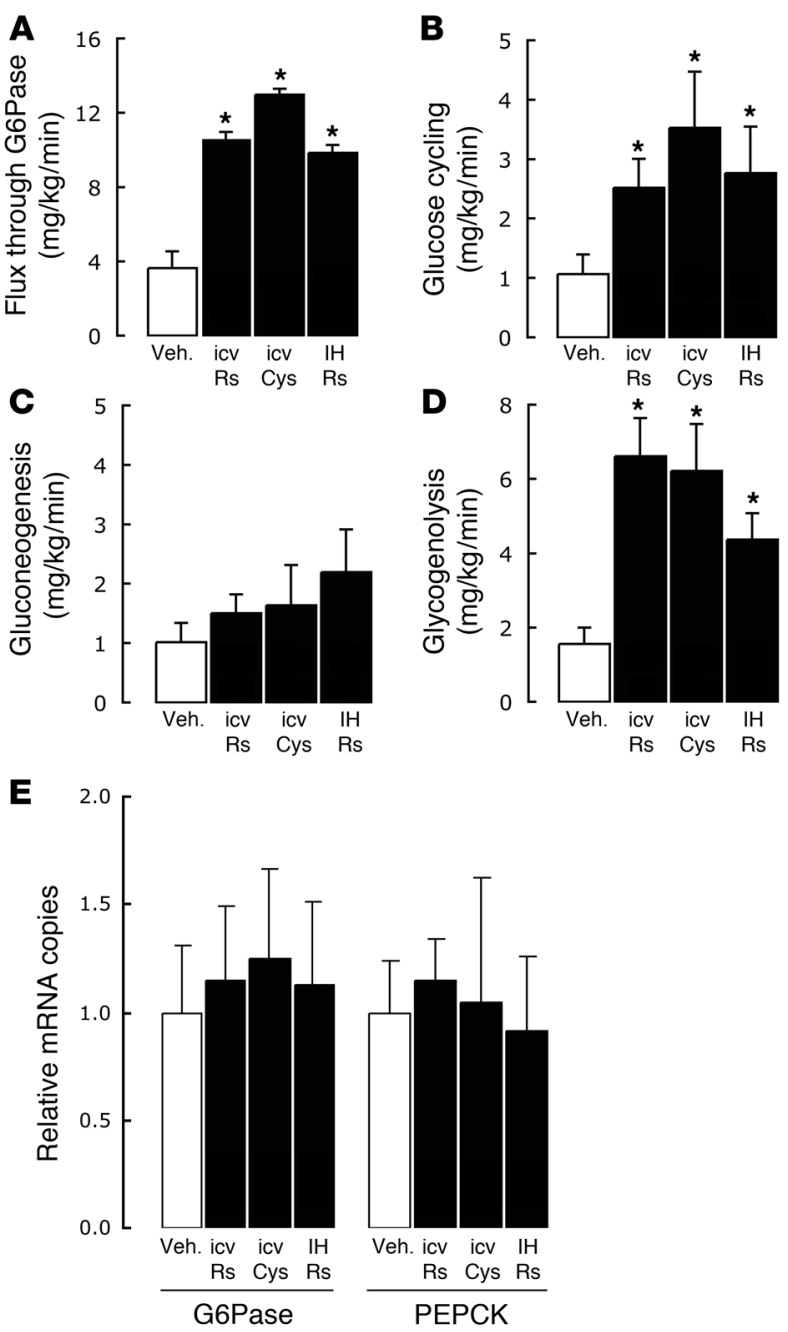

$\mathbf{F}$
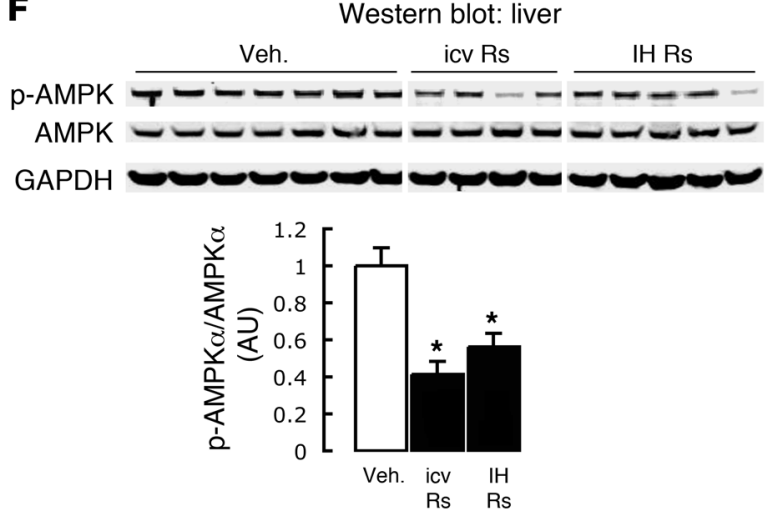

completely accounted for the effect of icv resistin or cys on wholebody glucose metabolism. Thus, a primary increase in central resistin action can stimulate GP in the presence of physiological levels of circulating insulin.

These potent metabolic effects of resistin could be mediated by its action anywhere within the CNS. To gain insight into the anatomical localization of these effects, we next infused a 20 -fold lower dose of resistin bilaterally within the MBH. MBH infusion of resistin entirely reproduced the effects of icv resistin on glucose

\section{Figure 2}

Central resistin increased hepatic glucoses fluxes predominantly via glycogenolytic pathways. (A and $\mathbf{B}$ ) icv or IH infusion of resistin (black bars) resulted in 2- to 3-fold increases in hepatic flux through G6Pase (A) and glucose cycling (B) compared with vehicle (white bars). (C and D) Increased hepatic glucose fluxes were mainly accounted for by an increased rate of glycogenolysis (D) rather than gluconeogenesis (C). (E) Quantitative real-time RT-PCR revealed that central resistin had no effect on the hepatic expression of the key gluconeogenic enzymes PEPCK and G6Pase. (F) Decreased levels of p-AMPK $\alpha$ were apparent in the livers of resistin-treated animals as analyzed by immunoblot. ${ }^{\star} P<0.05$ compared with vehicle.

infusion and GP (Figure 1, C-F). Thus, increased MBH availability of resistin is sufficient to increase GP.

GP represents the net contribution of glucosyl units derived from gluconeogenesis and glycogenolysis. However, a portion of glucose entering the liver via the direct phosphorylation of glucose is also a substrate for dephosphorylation via glucose6-phosphatase (G6Pase) creating a futile cycle, termed "glucose cycling". In order to further delineate the mechanisms by which central administration of resistin modulates glucose homeostasis, we estimated the in vivo flux of glucose through G6Pase and the contribution of gluconeogenesis and glycogenolysis relative to glucose output. icv resistin, icv cys, and $\mathrm{MBH}$ resistin markedly increased the glucose flux through G6Pase (Figure 2A) and glucose cycling (Figure 2B) in parallel with their effects on GP (Figure 1E). Importantly, the increase in GP was largely accounted for by a marked induction of glycogenolysis, while the rate of gluconeogenesis was only modestly increased (Figure 2, C and D). Realtime PCR analyses revealed that icv resistin, icv cys, and MBH resistin did not alter the hepatic mRNA levels for the rate-limiting enzymes for gluconeogenesis, G6Pase, and phosphoenolpyruvate (PEP) carboxykinase (PEPCK) (Figure 2E) On the other hand, both icv and $\mathrm{MBH}$ each resistin moderately decreased the hepatic levels of phosphorylated AMP-activated protein kinase $\alpha$ (p-AMPK $\alpha$ ) as analyzed by immunoblot (Figure $2 \mathrm{~F}$ ). Thus, direct enhancement of resistin action within the third cerebral ventricle or $\mathrm{MBH}$ per se was sufficient to recapitulate the action of systemic resistin on the in vivo fluxes through G6Pase, rates of glycogenolysis, and on the hepatic phosphorylation of AMPK.

As it is now widely accepted that increases in adiposity lead to an increased physiological inflammatory milieu, we examined the effect of central resistin on the expression of key inflammatory regulators in the liver (43-46). Strikingly, as determined by quantitative real-time RT-PCR, central resistin administration leads to an approximately 3 - to 4 -fold increase in the hepatic expression of SOCS- 3 and TNF- $\alpha$ mRNA and an even greater increase in the expression of IL-6 at the end of the clamps (Figure 3A). However, resistin infusion did not alter the expression of IKK- $\beta$ or the other hepatic gluco- and lipo-regulatory genes, PPAR $\gamma$ coactivator $1 \alpha$ (PGC-1 $\alpha$ ), fatty acid synthase (FAS), acetyl-CoA carboxylase 1 (ACC1), and steroyl-CoA decarboxylase 1 (SCD1) (Figure 3A). Mirroring the changes in gene expression and hepatic insulin action on glucose fluxes, immunoblot analysis revealed that hepatic SOCS-3 protein levels were similarly elevated. Phosphorylated glycogen synthase kinase $3 \beta$ (p-GSK3 $\beta$ ) was decreased, and the phosphorylation state of IкB- $\alpha$, a downstream target of IKK- $\beta$, was unchanged following central resistin administration (Figure 3, B and C). Finally, hepatic levels of STAT3 protein were dramati- 
A

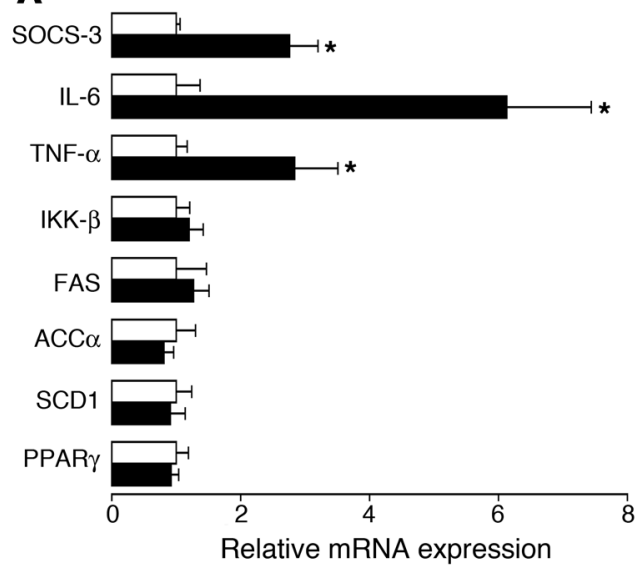

B

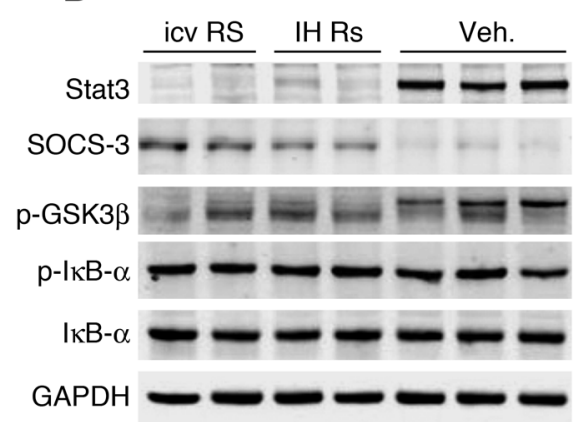

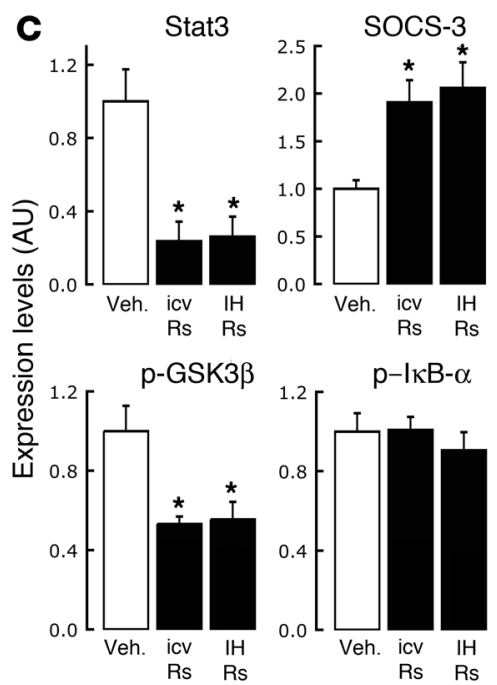

Figure 3

Real-time RT-PCR and Western immunoblot analysis of hepatic inflammation and insulin signaling. (A) icv resistin administration (black bars) increased hepatic gene expression of proinflammatory mediators SOCS-3, IL-6, and TNF- $\alpha$ compared with vehicle-treated animals (white bars) but had no change on IKK- $\beta$, FAS, ACC1, SCD1, and PPAR $\gamma$. (B and C) Significant decreases in total Stat3 protein and p-GSK3 $\beta$ were detected in the livers following central resistin administration (black bars), with a reciprocal but converse elevation in SOCS-3 when compared with controls (white bars). The levels of $\mathrm{p}-\mathrm{I \kappa} \mathrm{B}-\alpha$ remained unchanged $(\mathbf{C}) .{ }^{*} P<0.05$ compared with vehicle.

cally lower in animals infused with resistin either icv or in the $\mathrm{MBH}$ (Figure 3, B and C).

These gain-of-function experiments suggest that modulation of resistin action within the $\mathrm{MBH}$ is sufficient to markedly affect liver glucose homeostasis and selective inflammatory markers. However, the physiological role of hypothalamic resistin in the regulation of GP cannot be determined by these gain-of-function studies and thus requires loss-of-function experiments in the presence of physiological increases in the circulating resistin levels.

Antagonism of bypothalamic resistin action. Is the action of resistin within the hypothalamus required for the effect of circulating hyperresistinemia on GP? To address this question, we developed an experimental strategy aimed at antagonizing the central action of resistin in the presence of systemic elevations in plasma resistin concentrations. We reasoned that if hypothalamic resistin action is required for stimulation of GP by hyperresistinemia, negating the $\mathrm{MBH}$ effects of resistin should diminish the ability of resistin to enhance GP in the presence of a physiological elevation in the plasma resistin levels. To this end, we used Rs Abs to selectively impede the action of the infused recombinant mouse resistin within the $\mathrm{MBH}$ (Figure 4A). A subset of i.v. resistin-infused animals also received a $\mathrm{MBH}$ infusion of a control anti-human resistin $\mathrm{Ab}(\mathrm{Con} \mathrm{Ab})$, with which mouse resistin fails to maintain antigenicity. This group served as a control for nonspecific effects that central infusion of $\mathrm{Ab}$ may have had on GP. We performed pancreatic-hyperinsulinemic clamp studies (Figure 4A) designed to increase the plasma insulin concentrations by approximately 3 -fold above basal levels. Such an increase is normally associated with a significant stimulation of peripheral glucose disposal and a marked decrease in GP and therefore allows one to examine the effects of insulin on both the liver and peripheral tissues. The rate of glucose utilization during the clamp studies was not significantly affected by peripheral (i.v.) infusion of resistin regardless of the $\mathrm{MBH}$ administration of resistin antibodies (Figure 4B). How- ever, the inhibition of GP during the insulin clamp studies was improved by the $\mathrm{MBH}$ administration of Rs $\mathrm{Ab}$ but not Con $\mathrm{Ab}$ (Figure 4C). Thus, antagonism of $\mathrm{MBH}$ resistin action diminishes the stimulatory effect of systemic resistin on hepatic GP in the presence of physiological hyperinsulinemia.

Similarly, MBH infusion of Rs Ab attenuated the marked elevations in glucose flux through G6Pase and total glucose cycling that were induced by peripheral resistin infusion (Figure 5, A and B). Again these changes in hepatic glucose fluxes were accounted for mainly by changes in the glycogenolysis, although the peripheral infusion of resistin in the Con $\mathrm{Ab}$ cohort also led to a modest yet nonsignificant increase in gluconeogenesis (Figure 5, C and D). That a hypothalamus-mediated effect of resistin on hepatic glucose fluxes predominantly occurs via glycogenolytic pathways (Figure 2D) is reinforced by the attenuation of this effect upon central blockade of resistin action (Figure 5D). In contrast to the absence of a centrally mediated effect of resistin on the hepatic expression of key glucoregulatory genes, the approximately 3- to 4-fold increase in G6Pase expression brought about by peripheral (i.v.) resistin infusion appeared to be a direct result of resistin in the liver, as there was no attenuation by $\mathrm{MBH}$ Rs Ab (Figure 5E). Hepatic PEPCK mRNA expression remained unchanged. In accordance with the changes in GP, MBH Rs Ab attenuated the decrease in $\mathrm{p}$-AMPK $\alpha$ associated with i.v. resistin infusion (Figure 5F).

Strikingly, hepatic gene expression analysis revealed that the increases in IL- 6 and TNF- $\alpha$ mRNAs following resistin infusion were entirely due to centrally mediated effects of resistin, as $\mathrm{MBH}$ Rs Ab completely blocked the induction of these transcripts despite the ongoing peripheral hyperresistinemia (Figure 6A). The elevation of liver SOCS-3 expression, however, appears to be mediated both directly and indirectly, as intrahypothalamic (IH) Rs Ab was unable to decrease the elevations in mRNA or protein levels in the presence of i.v. resistin infusion (Figure 6, A-C). Resistin failed to demonstrate any direct or central action on the regula- 


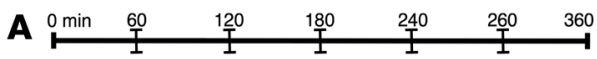

$\mathrm{IH}$ resistin antibody $(0.5 \mu \mathrm{l} / \mathrm{h})$

i.v. Resistin $(30 \mu \mathrm{g})$

[ $\left.{ }^{3} \mathrm{H}-3\right]$ glucose $(0.4 \mu \mathrm{Ci} / \mathrm{min})$

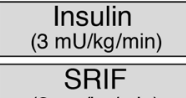

SRIF

Glucose

(as needed)
B

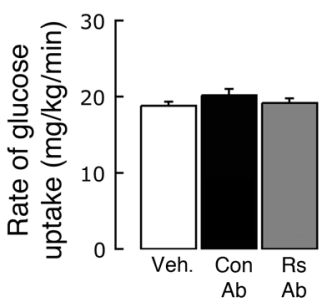

C

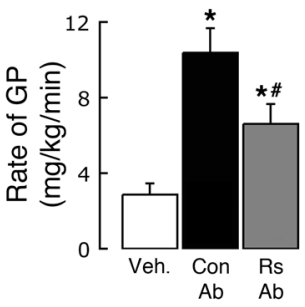

D

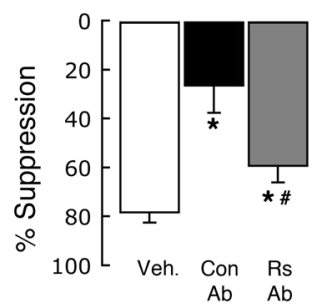

Figure 4

The effects of increased levels of circulating plasma resistin are attenuated in animals treated with IH anti-resistin antibodies. (A) Experimental design for hyperinsulinemic-euglycemic clamp studies. IH infusion of Con Ab or Rs Ab (0.33 $\mu$ l bolus followed $0.5 \mu \mathrm{l} / \mathrm{h}$ infusion) was initiated at 0 minutes and continued throughout the 360 -minute course of study. Resistin ( $30 \mu \mathrm{g}$ total dose) was infused i.v. at a constant rate starting at 60 minutes. The remainder of the study was completed as described in Figure 1. (B) Rates of glucose uptake during the insulin clamp studies were unaffected in all groups. (C) The rate of endogenous GP for i.v. resistin-infused animals also receiving an IH infusion of Con Ab was greatly increased compared with controls but was attenuated in animals receiving the $\mathrm{IH}$ infusion of $\mathrm{Rs} \mathrm{Ab}$. (D) Changes in the percentage suppression of endogenous GP in animals receiving i.v. resistin and IH Ab infusions. ${ }^{*} P<0.05$ compared with vehicle; ${ }^{\#} P<0.05$ compared with Con Ab.

tion of hepatic IKK- $\beta$, PGC-1 $\alpha$, FAS, ACC1, and SCD1 (Figure $6 \mathrm{~A})$. Blockade of central resistin action produced no alteration of GSK3 $\beta$ phosphorylation or total STAT3 levels, despite the increase in hepatic insulin action, thus suggesting potential effects regulated by both direct and indirect centrally mediated resistin action on these parameters (Figure 6, B and C).

\section{Discussion}

The glucoregulatory role of several adipokines such as leptin, adiponectin, and resistin is now well supported by genetic and physiological evidence (47). Specifically, bidirectional changes in the circulating levels of resistin induce robust changes in glucose homeostasis in rodents $(12,17-20,48)$. However, the observation that, in humans, circulating resistin is mostly derived from macrophages rather than adipocytes has suggested a divergence of physiological functions of this hormone in different species (16). In this regard, it has also been postulated that resistin constitutes an important link between innate immunity and glucose metabolism. This study extends our understanding of resistin physiology in 2 important ways. First is the discovery that resistin exerts its potent metabolic actions on the liver partly via its action within the hypothalamus. Second is the recognition that resistin exerts a proinflammatory influence on the liver through both peripheral and central mechanisms.

Resistin primarily exerts its glucoregulatory effect by stimulating hepatic glucose output (17-20). Our study confirms this, as we now show that an i.v. infusion of resistin $(30 \mu \mathrm{g})$ during the course of a 6-hour hyperinsulinemic-euglycemic clamp induces marked hepatic insulin resistance, with the effect being mainly accounted for by a failure of insulin to suppress basal levels of glycogenolysis. This elevation in peripheral resistin also recapitulated prior reports of the ability of resistin to reduce the phosphorylation state of both the fuel-sensing enzyme AMPK and GSK3 $\beta$, an important downstream target of the insulin signaling cascade, in the liver $(12,17,19)$. While the effect of resistin on insulin sensitivity in humans has been much debated $(21,49-52)$, it should be quite clear that elevation of circulating resistin in rodents, either acutely, such as in the current model, or chronically, such as in transgenic mice (20), following adenoviral-mediated overexpres- sion (48), or following diet-induced obesity (17), leads to marked decreases in hepatic insulin sensitivity.

The systemic infusion of resistin also resulted in the elevation of mRNA levels of several key inflammatory mediators (SOCS-3, TNF- $\alpha$, and IL-6) in the liver. Consistent with these findings, resistin-infused animals also exhibited elevations in SOCS-3 protein and a dramatic decrease in hepatic STAT3. As a result, one could view resistin as a potent mediator of both proinflammatory and insulin resistance pathways (44).

The CNS plays a major role in the regulation of whole-body physiology. In this regard, recent evidence suggests that nutritional and hormonal signals originating in the brain, especially within the hypothalamus, control glucose fluxes in insulin-sensitive organs (53). In particular, hormones that circulate in relation to body adiposity, such as leptin and insulin, exert some of their gluco-regulatory actions through the activation of hypothalamic receptors. Based on these previous observations, we postulated that the adipose-derived hormone resistin also exerts some of its metabolic actions through a central (hypothalamic) site of action. To this end, we first investigated whether a primary increase in hypothalamic resistin per se could regulate hepatic glucose fluxes. Indeed, infusion of recombinant mouse resistin directly into the third cerebral ventricle or within the $\mathrm{MBH}$ of conscious rats was sufficient to recapitulate the systemic effects of resistin not only on hepatic GP but also on inflammatory mediators. This effect was verified using an additional preparation of resistin, cys, which exists only as a trimer but has previously been shown to retain full biological activity (42). Of interest, the central administration of resistin markedly and selectively impaired the inhibitory action of insulin on hepatic glycogenolysis but failed to alter the hepatic expression of the key gluconeogenic enzymes PEPCK and G6Pase. This argues against a resistin-mediated antagonism of insulin action at the level of the hypothalamus, as intact central insulin action has been shown to be required for an insulininduced decrease in the rate of hepatic gluconeogenesis, an effect clearly retained in these experiments (54). Thus, it is plausible that the hypothalamus-mediated effect of resistin on hepatic glucose flux impairs the direct (but not the hypothalamic) effects of insulin action on liver glucose fluxes. Hypothalamic resistin 
A

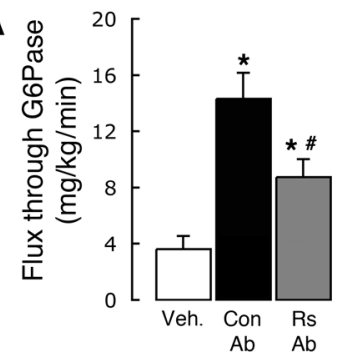

C

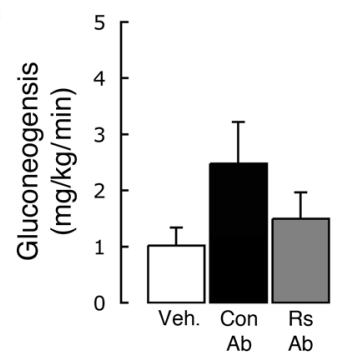

E

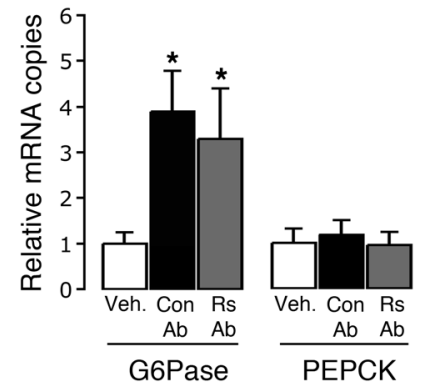

$\mathbf{F}$
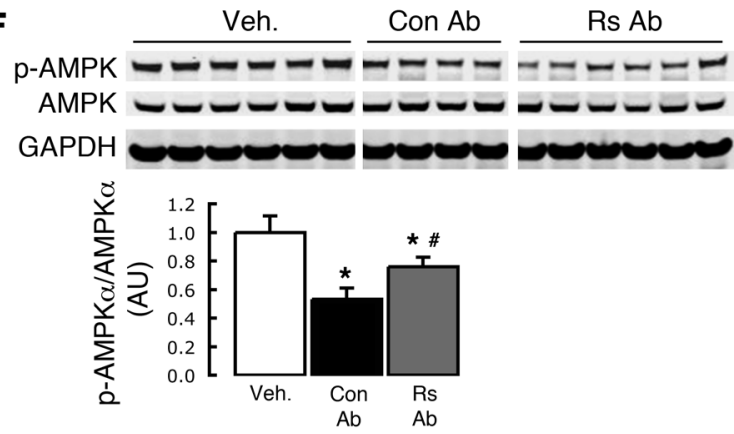

administration also recapitulated the effect of peripheral resistin on hepatic p-AMPK levels and completely mirrored the effects of its systemic administration on the hepatic gene expression of SOCS-3, TNF- $\alpha$, and IL- 6 .

The physiological relevance of this effect of central resistin on hepatic GP, which we believe to be novel, is clearly illustrated by the use of blocking antibodies against resistin at the level of the $\mathrm{MBH}$ in the presence of elevated circulating resistin levels following i.v. resistin infusion. It should be pointed out, however, that our loss-of-function central blockade experiments should be considered a minimal estimate of the central effect of resistin. There are several reasons for this. First, the efficiency of blockade provided by the $\mathrm{Ab}$ infusions remains undetermined. It is likely that the IH Ab infusion failed to completely block resistin signaling within the MBH. Second, other areas of the CNS could possibly play a role in the central effects of resistin. We have shown that the icv administration of resistin causes robust effects in terms of

\section{Figure 5}

Central blockade of resistin after peripheral (i.v.) resistin infusion attenuates increases in hepatic glucose fluxes. (A and $\mathbf{B}$ ) IH administration of Rs Ab decreased hepatic flux through G6Pase (A) and glucose cycling (B) in animals receiving a constant i.v. infusion of resistin, compared with animals receiving $\mathrm{Con} \mathrm{Ab}$, although it failed to completely normalize these to vehicle-treated levels. (C and D) Alterations of hepatic glucose fluxes in these animals were mirrored by concomitant changes in glycogenolysis (D) but not gluconeogenesis (C). (E) Peripheral (i.v.) infusion of resistin increased the hepatic expression of G6Pase message levels, which were not attenuated by central Rs $\mathrm{Ab}$ administration as assayed by real-time RT-PCR. Parallel changes in PEPCK expression levels were absent. (F) Depressed levels of p-AMPK $\alpha$ and subsequent, albeit minor, attenuation of this effect by central Rs Ab were apparent in the livers of i.v. resistin-treated animals compared with vehicle as analyzed by immunoblot. ${ }^{*} P<0.05$ compared with vehicle; $\#<0.05$ compared with Con $\mathrm{Ab}$.

regulating hepatic glucose fluxes as well as altering proinflammatory gene expression profiles, but other brain regions with direct connections to the ventricular system may also mediate the actions of resistin. The $\mathrm{Ab}$ infusions were only directed into the $\mathrm{MBH}$ such that resistin signaling could have been activated in other areas within the CNS. Therefore, the results from our loss-of-function Ab blockade studies must be interpreted as providing an estimate of the minimal contribution of hypothalamic resistin to the physiological effects of circulating resistin.

The infusion of $\mathrm{Rs} \mathrm{Ab}$ but not Con $\mathrm{Ab}$ into the MBH reduced the effect of resistin on the rate of hepatic GP by approximately $50 \%$. This blunting was not only apparent in the calculated rates of GP but was also mirrored by the parallel changes in hepatic glucose fluxes, including the rate of glycogenolysis. As such, resistin appeared to modulate hepatic insulin sensitivity through both direct action on the liver and through hypothalamus-mediated mechanisms that account for at least $50 \%$ of the effect of resistin on hepatic glucose fluxes. Central blockade of resistin signaling within the $\mathrm{MBH}$ also almost completely abolished the hepatic proinflammatory response. On the other hand, $\mathrm{MBH}$ blockade of resistin action failed to restore hepatic AMPK phosphorylation. Therefore, it is likely that resistin regulates hepatic AMPK activity through redundant peripheral and central mechanisms. Importantly, the central administration of resistin or of $\mathrm{Rs} \mathrm{Ab}$ failed to alter the hepatic expression of G6Pase. These results indicate that the ability of resistin to increase G6Pase mRNA expression is independent of its $\mathrm{MBH}$ effects. In agreement with prior reports, we show that SOCS-3 gene expression and protein levels are increased following resistin treatment (55). However, a central blockade of resistin action in the $\mathrm{MBH}$ was unable to counter the increases of hepatic SOCS-3 message levels induced by peripheral i.v. resistin administration despite the increase in hepatic insulin action. Thus, while resistin appears to have an impact on hepatic SOCS-3 mRNA and protein levels both indirectly via the $\mathrm{MBH}$ and directly on the liver itself, these elevations in SOCS-3 may be disconnected from the acute effects of central resistin on hepatic insulin sensitivity. While it is well known that the SOCS proteins SOCS-1 and SOCS-3 are implicated in hepatic insulin resistance (30), it remains to be established whether the elevations of SOCS-3 that are seen after central and peripheral resistin administration are necessary and required for mediating the effects of resistin on hepatic insulin sensitivity.

We propose that this hypothalamus-mediated effect of resistin on promoting an increased inflammatory milieu may prove to be 
A

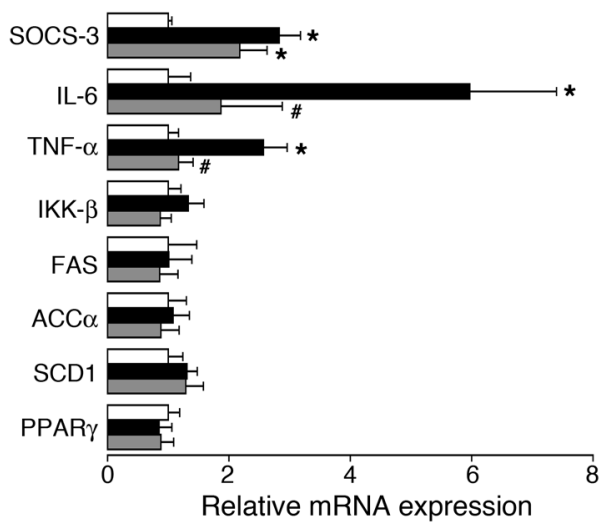

B

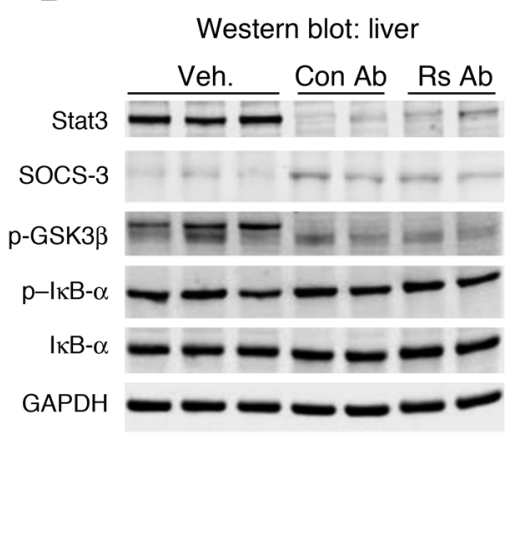

C

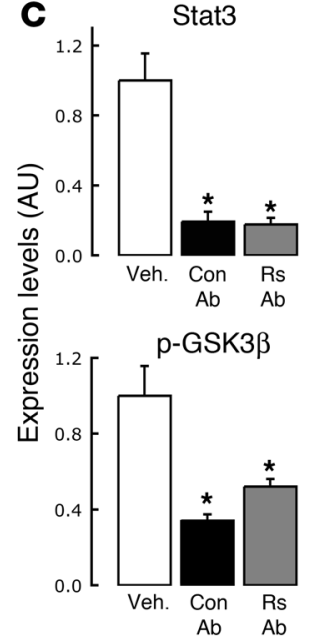

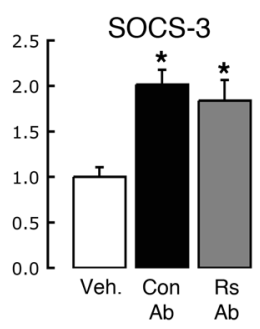

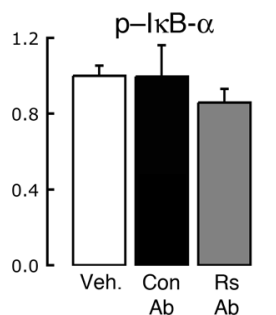

Figure 6

The increased expression of inflammatory genes in the liver following peripheral (i.v.) resistin infusion is ameliorated by central Rs Ab. (A) The increase in hepatic gene expression of key inflammatory mediators TNF- $\alpha$ and IL- 6 in animals receiving a peripheral resistin infusion ( $30 \mu \mathrm{g}$ total over $5 \mathrm{~h}$ ) with central $(\mathrm{IH})$ Con Ab (black bar) compared with vehicle-infused animals (white bars) was attenuated in animals receiving the same peripheral resistin infusion ( $30 \mu \mathrm{g}$ total over $5 \mathrm{~h}$ ) but with IH Rs Ab treatment (gray bars), blockade of central resistin signaling was unable to repress SOCS-3 expression. No change on IKK- $\beta$, FAS, ACC $\alpha$, SCD1, or PPAR $\gamma$ gene expression was noticed following peripheral resistin infusion in either group. (B and C) Parallel to changes in hepatic gene expression, SOCS-3 protein levels as analyzed by Western blot were elevated following i.v. resistin infusion, with no amelioration of this effect by central Rs Ab. The decrease of hepatic STAT3 protein and p-GSK3 $\beta$ levels also remained unaffected by central resistin blockade. No changes in $p-l \kappa B-\alpha$ were detected following peripheral resistin administration in either cohort (black and gray bars) compared with vehicle (white bars). ${ }^{*} P<0.05$ compared with vehicle; ${ }^{\#} P<0.05$ compared with Con Ab.

crucial in the effects of resistin in human pathophysiology. The growing evidence of a role for resistin in innate immunity provides a common basis for understanding the hypothetical function of this hormone in rodents and in humans independent of its point of origin (in adipocytes and monocytes/macrophages, respectively). It is entirely plausible that the contribution of resistin within the CNS to promoting and maintaining an inflammatory environment, which over time leads to decreased insulin sensitivity, could become relevance in humans and be regarded as major factor in the development of insulin resistance, atherosclerosis, and hyperlipidemia associated with the metabolic syndrome in adults with increased adiposity.

\section{Methods}

Recombinant resistin. Recombinant resistin was purified as previously described by Rajala et al. (18), with some modifications. In brief, the entire open reading frame of resistin was cloned into pFM1, which contains an internal ribosome entry site followed by the open reading frame of GFP, as previously described. Stably transfected HEK 293 T cells were FACS sorted for high levels of GFP expression and therefore high resistin expression. After sorting 5 times for enrichment, we established a line of cells capable of producing milligram quantities of recombinant protein per liter of media. Serum-free media was used to collect the secreted protein from confluent cells for 2 days. The media was then harvested, spun down to remove cells, and $20 \mathrm{mM}$ Bis-Tris $\mathrm{pH} 6.0$ used to adjust the $\mathrm{pH}$ to 6.0. The media was then filtered with a $0.22-\mathrm{mm}$ filter and precipitated with $40 \%$ ammonium sulfate. Precipitated material was brought up in loading buffer (20 mM Bis-Tris, $25 \mathrm{mM} \mathrm{NaCl}$, pH 6.0) and run over a HiPrep 26/10 Desalting column (GE Healthcare) to equilibrate the protein in loading buffer. Protein was then loaded onto a Hitrap SP HP ion exchange column (GE Healthcare), and a gradient elution was performed to $1 \mathrm{M} \mathrm{NaCl}$.
A single peak was apparent that was eluted between 350 and $450 \mathrm{mM} \mathrm{NaCl}$. This eluate was then concentrated by centrifugation using an Amicon Ultra with a MW cut-off of $30 \mathrm{kDa}$ (Millipore), and the resulting concentrate was loaded onto a HiPrep 26/10 Desalting column (GE Healthcare) to equilibrate the protein in $1 \times$ PBS. The major peak off the ion exchange column contained at least $98 \%$ pure recombinant protein as judged by a Coomassie Blue-stained SDS-PAGE gel analysis (Invitrogen).

Animals. We housed 10-week-old male Sprague Dawley rats (Charles River Laboratories) in individual cages in a temperature- and light-controlled (12-hour light/12-hour dark cycle) facility. Animals were stereotaxically fit with indwelling icv (coordinates from bregma: A/P $+0.2 \mathrm{~mm}, \mathrm{D} / \mathrm{V}$ $-9.0 \mathrm{~mm}$ ) or IH (coordinates from bregma: A/P $-3.3 \mathrm{~mm}, \mathrm{D} / \mathrm{V}-10.5 \mathrm{~mm}$ ) cannulae (Plastics One Inc.) 2 weeks prior to hyperinsulinemic-euglycemic clamp study, followed 1 week later by intracarotid arterial and intrajugular venous catheterization for i.v. infusion and blood sampling, respectively. Animals were allowed to recover for 5 days following the final surgery.

Hyperinsulinemic-englycemic clamp studies. For in vivo studies, rats were food restricted $(20 \mathrm{~g})$ on the night prior to experimentation. At 0 minutes, either an icv or IH infusion of artificial cerebrospinal fluid (aCSF) or resistin (wild-type or cys), or the IH infusion of resistin $\mathrm{Ab}$ was initiated, depending on the study. For animals that received IH anti-resistin antibodies, an i.v. infusion of resistin was started at 60 minutes and run continuously until the end of the study. A primed-continuous infusion of $\left[{ }^{3} \mathrm{H}-3\right]$-glucose (New England Nuclear; $40 \mu \mathrm{Ci}$ bolus; $0.4 \mu \mathrm{Ci} / \mathrm{min}$ ) was initiated at 120 minutes and was maintained throughout the study to assess glucose kinetics. Samples for the determination of $\left[{ }^{3} \mathrm{H}\right]$-glucose specific activity were obtained at 10-minute intervals. A hyperinsulinemic-euglycemic clamp was performed during the final 2 hours $(240-360 \mathrm{~min})$ of the study. In brief, a continuous infusion of insulin $(3 \mathrm{mU} / \mathrm{kg} / \mathrm{min})$ and somatostatin $(3 \mu \mathrm{g} / \mathrm{kg} / \mathrm{min})$ was administered, and a variable infusion of a $25 \%$ glucose solution was started and periodically adjusted to clamp and maintain the 
plasma glucose concentration at approximately $8 \mathrm{mM}$. Ten minutes before the end of the infusion studies, $\left[\mathrm{U}-{ }^{14} \mathrm{C}\right]$-lactate (New England Nuclear; 20 $\mu \mathrm{Ci}$ bolus; $1.0 \mu \mathrm{Ci} / \mathrm{min}$ ) was administered to determine the contribution of gluconeogenesis to the pool of hepatic glucose-6-phosphate. At the end of the infusion experiments, rats were anesthetized, animal tissues were freeze-clamped in situ with aluminum tongs that had been precooled in liquid nitrogen immediately upon sacrifice, and tissues were flash frozen in liquid nitrogen before storing at $-80^{\circ} \mathrm{C}$.

Analytical procedures. Plasma glucose was measured by glucose oxidase (Glucose 2 Analyzer; Beckman Coulter). Under steady-state conditions for plasma glucose concentration, the rate of glucose disappearance (Rd) equals the rate of glucose appearance $(\mathrm{Ra})$. $\mathrm{Ra}$ was determined from the ratio of the infusion rate for $\left[{ }^{3} \mathrm{H}\right]$ glucose (disintegrations per minute) and the specific activity of plasma $\left[{ }^{3} \mathrm{H}\right]$ glucose (disintegrations per minute per milligram glucose) under steady-state conditions. The rate of GP was therefore obtained from the difference between $\mathrm{Ra}$ and the rate of glucose infusion. The hepatic $\left[{ }^{14} \mathrm{C}\right] \mathrm{PEP}$ and $\left[{ }^{3} \mathrm{H} /{ }^{14} \mathrm{C}\right] \mathrm{UDP}$-glucose-specific activities (UDP-glucose, uridine diphospho-glucose) were measured by HPLC, and the rates of PEP gluconeogenesis were calculated. The percentage of the hepatic glucose-6-phosphate pool directly derived from plasma glucose (direct pathway) was calculated as the ratio of liver $\left[{ }^{3} \mathrm{H}\right] \mathrm{UDP}$-glucose and plasma $\left[{ }^{3} \mathrm{H}-3\right]$ glucose-specific activities. Gluconeogenesis was estimated from the specific activities of ${ }^{14} \mathrm{C}$-labeled hepatic UDP-glucose (assumed to reflect the specific activity of hepatic glucose-6-phosphate), and hepatic PEP following the infusion of $\left[\mathrm{U}-{ }^{14} \mathrm{C}\right]$ lactate and $\left[{ }^{3} \mathrm{H}-3\right]$ glucose using the following formula: $\mathrm{GNG}=\mathrm{TGO} \times\left[{ }^{14} \mathrm{C}\right] \mathrm{UDP}$-glucose SA $/\left[{ }^{14} \mathrm{C}\right] \mathrm{PEP} \mathrm{SA} \times 2$ (GNG, gluconeogenesis; SA, specific activity; TGO, total glucose output).

$R N A$ extraction and quantitative real-time RT-PCR. Total liver RNA was obtained from frozen tissue (approximately $85-100 \mathrm{mg}$ ) using TRIzOL reagent (Invitrogen) according to the manufacturer's instructions. Following treatment with DNase I (Invitrogen), purified RNA was used as template for first-strand cDNA synthesis using SuperScript III (Invitrogen). Quantitative real-time RT-PCR was run using LC-Fast Start DNA SYBR Green I chemistry (Roche Diagnostics) on a LightCycler 2.0 platform (Roche Diagnostics). Forward and reverse primer pairs were as listed in Supplemental Table 3.

Western blot analyses. Liver tissues were homogenized in $20 \mathrm{mM}$ Mops, $2 \mathrm{mM}$ EGTA, $5 \mathrm{mM}$ EDTA, $30 \mathrm{mM}$ sodium fluoride, $40 \mathrm{mM} \beta$-glycerophosphate, $10 \mathrm{mM}$ sodium pyrophosphate, $2 \mathrm{mM}$ orthovanadate, $0.5 \% \mathrm{NP}-40$, Complete phosphatase inhibitor cocktail (Roche). Protein concentration was measured with a BCA protein quantification kit (Pierce Biotechnology). Protein extracts were run on either Criterion XL 4\%-12\% (Bio-Rad) or $4 \%-12 \%$ Bis-Tris NuPAGE (Invitrogen) gels and were blotted onto Immobilon FL PVDF (Millipore). Immunoblots were blocked at room temperature for 1 hour in Odyssey LI-COR Blocking Buffer (LI-COR Biosciences) and incubated in primary Abs against p-AMPK $\alpha$, AMPK $\alpha$, p-Akt473, Akt, p-GSK3 $\beta$, p-STAT3-705 (Cell Signaling Technology), SOCS-3, STAT3, IкB- $\alpha$, p-IкB- $\alpha$ (Santa Cruz Biotechnology Inc.) and GAPDH (Research Diagnostics Inc.) in 1:1 blocking buffer/Tris-buffered saline Tween 20 (TBST) over- night at $4^{\circ} \mathrm{C}$. Following 3 consecutive 5 -minute washes in TBST $(0.1 \%)$, blots were incubated with Alexa Fluor 680-conjugated donkey anti-goat IgG, Alexa Fluor 680-conjugated anti-mouse IgG (Invitrogen), or IR Dye 800-conjugated goat anti-rabbit IgG (Rockland Immunochemicals) for 1 hour at room temperature in blocking buffer containing $0.1 \%$ TBST and $0.1 \%$ SDS. After 2 washes in TBST and a final wash in TBS, the blots were scanned using the Odyssey Infrared Imaging System (LI-COR Biosciences) (LI-COR Biosciences) and quantified using Odyssey 2.0 software based on direct fluorescence measurement.

Statistics. All values are presented as the mean \pm SEM. Comparisons among groups were made using ANOVA followed by unpaired, nonparametric 2-tailed Student's $t$ test. Differences were considered statistically significant at $P<0.05$. The study protocol was reviewed and approved by the Institutional Animal Care and Use Committee of the Albert Einstein College of Medicine.

\section{Acknowledgments}

We wish to thank Bing Liu, Clive Baveghems, and Stanislaw Gaweda for expert technical assistance; Michael Rajala for the generation of the resistin-producing HEK 293 T cells; and Todd Schraw for providing purified resistin. The authors also appreciate the careful review of this manuscript by Raul C. Camacho and Andrea R. Nawrocki. This work was supported by grants to L. Rossetti from the NIH (R01-DK45024 and R37-DK48321) and to P.E. Scherer (R01-DK55758, R01-CA112023, and R21-DK075887). Furthermore, P.E. Scherer and L. Rossetti have joint grant support from R24-DK071030 and the Core Laboratories of the Albert Einstein Diabetes Research and Training Center (P60-DK 20541). P.E. Scherer is also a recipient of an Irma T. Hirschl Career Scientist Award. E.D. Muse is the recipient of a Physician Scientist Training Award from the American Diabetes Association. T.K.T. Lam was supported by a fellowship from the NIH (F32-DK072876).

Received for publication September 22, 2006, and accepted in revised form April 10, 2007.

Address correspondence to: Luciano Rossetti, Department of Pharmacology and Diabetes Research and Training Center, Albert Einstein College of Medicine, 1300 Morris Park Avenue, Bronx, New York 10461, USA. Phone: (718) 430-4118; Fax: (718) 4308557; E-mail: rossetti@aecom.yu.edu.

Tony K.T. Lam's present address is: Departments of Physiology and Medicine, University Health Network and University of Toronto, Toronto, Ontario, Canada.

Philipp E. Scherer's present address is: Touchstone Diabetes Center, Department of Internal Medicine, University of Texas Southwestern Medical Center, Dallas, Texas, USA.
1. Adams, K.F., et al. 2006. Overweight, obesity, and mortality in a large prospective cohort of persons 50 to 71 years old. N. Engl. J. Med. 355:763-778.

2. Ogden, C.L., et al. 2006. Prevalence of overweight and obesity in the United States, 1999-2004. JAMA. 295:1549-1555.

3. Kahn, B.B., and Flier, J.S. 2000. Obesity and insulin resistance. J. Clin. Invest. 106:473-481.

4. Flier, J.S. 2004. Obesity wars: molecular progress confronts an expanding epidemic. Cell. 116:337-350.

5. Boden, G., et al. 2005. Free fatty acids produce insulin resistance and activate the proinflammatory nuclear factor-kappaB pathway in rat liver. Diabetes.
54:3458-3465.

6. Berg, A.H., Combs, T.P., Du, X., Brownlee, M., and Scherer, P.E. 2001. The adipocyte-secreted protein Acrp30 enhances hepatic insulin action. Nat. Med. 7:947-953.

7. Kadowaki, T., et al. 2006. Adiponectin and adiponectin receptors in insulin resistance, diabetes, and the metabolic syndrome. J. Clin. Invest. 116:1784-1792. doi:10.1172/JCI29126.

8. Xu, H., et al. 2003. Chronic inflammation in fat plays a crucial role in the development of obesity-related insulin resistance. J. Clin. Invest. 112:1821-1830. doi:10.1172/JCI200319451.
9. Wellen, K.E., and Hotamisligil, G.S. 2005. Inflammation, stress, and diabetes. J. Clin. Invest. 115:1111-1119. doi:10.1172/JCI200525102.

10. Rondinone, C.M. 2006. Adipocyte-derived hormones, cytokines, and mediators. Endocrine. 29:81-90.

11. McTernan, P.G., Kusminski, C.M., and Kumar, S. 2006. Resistin. Curr. Opin. Lipidol. 17:170-175.

12. Steppan, C.M., et al. 2001. The hormone resistin links obesity to diabetes. Nature. 409:307-312.

13. Holcomb, I.N., et al. 2000. FIZZ1, a novel cysteinerich secreted protein associated with pulmonary inflammation, defines a new gene family. EMBOJ. 19:4046-4055. 
14. Steppan, C.M., et al. 2001. A family of tissue-specific resistin-like molecules. Proc. Natl. Acad. Sci. U. S. A. 98:502-506.

15. Savage, D.B., et al. 2001. Resistin/Fizz3 expression in relation to obesity and peroxisome proliferator-activated receptor-gamma action in humans. Diabetes. 50:2199-2202.

16. Patel, L., et al. 2003. Resistin is expressed in human macrophages and directly regulated by PPAR gamma activators. Biochem. Biophys. Res. Commun. 300:472-476

17. Muse, E.D., et al. 2004. Role of resistin in dietinduced hepatic insulin resistance. J. Clin. Invest. 114:232-239. doi:10.1172/JCI200421270.

18. Rajala, M.W., Obici, S., Scherer, P.E., and Rossetti, L. 2003. Adipose-derived resistin and gut-derived resistin-like molecule- $\beta$ selectively impair insulin action on glucose production. J. Clin. Invest. 111:225-230. doi:10.1172/JCI200316521.

19. Banerjee, R.R., et al. 2004. Regulation of fasted blood glucose by resistin. Science. 303:1195-1198.

20. Rangwala, S.M., et al. 2004. Abnormal glucose homeostasis due to chronic hyperresistinemia. Diabetes. 53:1937-1941.

21. McTernan, P.G., et al. 2002. Increased resistin gene and protein expression in human abdominal adipose tissue. J. Clin. Endocrinol. Metab. 87:2407.

22. Silha, J.V., et al. 2003. Plasma resistin, adiponectin and leptin levels in lean and obese subjects: correlations with insulin resistance. Eur. J. Endocrinol. 149:331-335.

23. Vendrell, J., et al. 2004. Resistin, adiponectin, ghrelin, leptin, and proinflammatory cytokines: relationships in obesity. Obes. Res. 12:962-971.

24. Reilly, M.P., et al. 2005. Resistin is an inflammatory marker of atherosclerosis in humans. Circulation. 111:932-939.

25. Gomez-Ambrosi, J., and Fruhbeck, G. 2001. Do resistin and resistin-like molecules also link obesity to inflammatory diseases? Ann. Intern. Med. 135:306-307.

26. Shoelson, S.E., Lee, J., and Goldfine, A.B. 2006 Inflammation and insulin resistance. J. Clin. Invest. 116:1793-1801. doi:10.1172/JCI29069.

27. Uysal, K.T., Wiesbrock, S.M., Marino, M.W., and Hotamisligil, G.S. 1997. Protection from obesityinduced insulin resistance in mice lacking TNFalpha function. Nature. 389:610-614.
28. Borst, S.E. 2004. The role of TNF-alpha in insulin resistance. Endocrine. 23:177-182.

29. Emanuelli, B., et al. 2000. SOCS-3 is an insulininduced negative regulator of insulin signaling. J. Biol. Chem. 275:15985-15991.

30. Ueki, K., Kondo, T., and Kahn, C.R. 2004. Suppressor of cytokine signaling 1 (SOCS-1) and SOCS-3 cause insulin resistance through inhibition of tyrosine phosphorylation of insulin receptor substrate proteins by discrete mechanisms. Mol. Cell. Biol. 24:5434-5446.

31. Inoue, H., et al. 2004. Role of STAT-3 in regulation of hepatic gluconeogenic genes and carbohydrate metabolism in vivo. Nat. Med. 10:168-174.

32. Cai, D., et al. 2005. Local and systemic insulin resistance resulting from hepatic activation of IKK-beta and NF-kappaB. Nat. Med. 11:183-190.

33. Morash, B.A., Willkinson, D., Ur, E., and Wilkinson, M. 2002. Resistin expression and regulation in mouse pituitary. FEBS Lett. 526:26-30.

34. Wilkinson, M., Wilkinson, D., Wiesner, G., Morash, B., and Ur, E. 2005. Hypothalamic resistin immunoreactivity is reduced by obesity in the mouse: co-localization with alpha-melanostimulating hormone. Neuroendocrinology. 81:19-30.

35. Brunetti, L., et al. 2004. Resistin, but not adiponectin, inhibits dopamine and norepinephrine release in the hypothalamus. Eur. J. Pharmacol. 493:41-44.

36. Obici, S., Zhang, B.B., Karkanias, G., and Rossetti, L. 2002. Hypothalamic insulin signaling is required for inhibition of glucose production. Nat. Med. 8:1376-1382

37. Friedman, J.M. 2000. Obesity in the new millennium. Nature. 404:632-634

38. Plum, L., Belgardt, B.F., and Bruning, J.C. 2006. Central insulin action in energy and glucose homeostasis. J. Clin. Invest. 116:1761-1766. doi:10.1172/ JCI29063.

39. Lam, T.K., Gutierrez-Juarez, R., Pocai, A., and Rossetti, L. 2005. Regulation of blood glucose by hypothalamic pyruvate metabolism. Science. 309:943-947.

40. Pocai, A., et al. 2006. Restoration of hypothalamic lipid sensing normalizes energy and glucose homeostasis in overfed rats. J. Clin. Invest. 116:1081-1091. doi:10.1172/JCI26640.

41. Lam, T.K., Schwartz, G.J., and Rossetti, L. 2005. Hypothalamic sensing of fatty acids. Nat. Neurosici. 8:579-584.
42. Patel, S.D., Rajala, M.W., Rossetti, L., Scherer, P.E., and Shapiro, L. 2004. Disulfide-dependent multimeric assembly of resistin family hormones. Science. 304:1154-1158.

43. Wellen, K.E., and Hotamisligil, G.S. 2003. Obesity-induced inflammatory changes in adipose tissue. J. Clin. Invest. 112:1785-1788. doi:10.1172/ JCI200320514.

44. Lehrke, M., et al. 2004. An inflammatory cascade leading to hyperresistinemia in humans. PLoS Med. 1:e45.

45. Bokarewa, M., Nagaev, I., Dahlberg, L., Smith, U., and Tarkowski, A. 2005. Resistin, an adipokine with potent proinflammatory properties. J. Immunol. 174:5789-5795.

46. Fu, Y., Luo, L., Luo, N., and Garvey, W.T. 2006. Proinflammatory cytokine production and insulin sensitivity regulated by overexpression of resistin in 3T3-L1 adipocytes. Nutr. Metab. (Lond.). 3:28.

47. Vettor, R., Milan, G., Rossato, M., and Federspil, G. 2005. Review article: adipocytokines and insulin resistance. Aliment. Pharmacol. Ther. 22(Suppl. 2):3-10.

48. Satoh, H., et al. 2004. Adenovirus-mediated chronic "hyper-resistinemia" leads to in vivo insulin resistance in normal rats. J. Clin. Invest. 114:224-231. doi:10.1172/JCI200420785.

49. Janke, J., Engeli, S., Gorzelniak, K., Luft, F.C., and Sharma, A.M. 2002. Resistin gene expression in human adipocytes is not related to insulin resistance. Obes. Res. 10:1-5.

50. Azuma, K., et al. 2003. Correlation between serum resistin level and adiposity in obese individuals. Obes. Res. 11:997-1001.

51. Nagaev, I., and Smith, U. 2001. Insulin resistance and type 2 diabetes are not related to resistin expression in human fat cells or skeletal muscle. Biochem. Biophys. Res. Commun. 285:561-564.

52. Stumvoll, M., and Haring, H. 2002. Resistin and adiponectin - of mice and men. Obes. Res. 10:1197-1199.

53. Schwartz, M.W., and Porte, D., Jr. 2005. Diabetes, obesity, and the brain. Science. 307:375-379.

54. Pocai, A., et al. 2005. Hypothalamic K(ATP) channels control hepatic glucose production. Nature. 434:1026-1031.

55. Steppan, C.M., Wang, J., Whiteman, E.L., Birnbaum, M.J., and Lazar, M.A. 2005. Activation of SOCS-3 by resistin. Mol. Cell. Biol. 25:1569-1575. 